\title{
In memoriam : Jacques E. Astier, 1923-2012
}

Jacques Astier est né à Dijon, le 3 janvier 1923. La mort de ses parents alors qu'il était encore très jeune l'a amené à être élevé par une tante, elle-même dijonnaise. Son enfance et sa prime jeunesse se déroulent dans cette ville et il en gardera toute sa vie un profond attachement à cette région de Bourgogne où il choisira de se trouver une résidence secondaire puis de se retirer lors de sa retraite.

Il est reçu au concours de 1942 à l'École Centrale de Paris. Toutefois son intégration à l'école sera retardée par le contexte de cette époque de guerre : Jacques, atteint de la tuberculose, est orienté au sanatorium de St Hilaire du Touvet, dans la Chartreuse. Ce séjour et le lieu où il s'accomplissait lui permettent d'entrer en rapport avec la Résistance et les futurs acteurs de la libération de Grenoble à laquelle il participera.

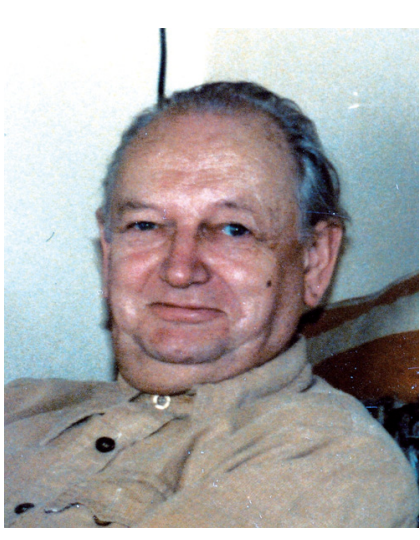

En attendant de rejoindre Centrale à la rentrée de 1945, Jacques s'inscrira pendant un an à la faculté de Physique de Dijon : c'est lors de cette période universitaire qu'il rencontrera Simone, sa future femme.

À sa sortie de l'École Centrale en 1948, Jacques Astier, recruté par Henri Malcor, est entré à l'IRSID (Institut de Recherches de la Sidérurgie) où il devait faire toute sa carrière. Le président Malcor travaillait alors personnellement à la création de services chargés de l'étude de l'élaboration de l'acier, au-delà des laboratoires qui avaient constitué la première raison de la création de l'IRSID.

Il fallait alors beaucoup de coke pour fondre au haut fourneau les minerais pauvres qui constituaient la seule ressource nationale en la matière. Un service « coke et fonte » était créé et installé à Longwy, chargé d'étudier les hauts fourneaux et de tenter d'améliorer leurs performances ; il devait être complété par un service Minerais qui étudierait la préparation des minerais et notamment leurs possibilités d'enrichissement par des méthodes physiques, ainsi que les procédés qui permettraient de passer du minerai à l'acier sans requérir l'emploi de cokes coûteux. Ce service n'existait pas encore quand Jacques Astier fut recruté, et il a été décidé de l'envoyer pendant une année aux États-Unis pour étudier la réduction en four tournant d'un minerai français, en l'espèce celui de Soumont en Normandie. L'avantage attendu des fours tournants était qu'ils pouvaient s'accommoder de combustibles bien moins coûteux que le coke. Aux États-Unis le Bureau of Mines disposait d'un four qui travaillait à basse température et semblait bien adapté à nos minerais. Encore fallait-il s'en assurer.

Jacques Astier et sa jeune épouse Simone partirent donc pour un an à Laramie dans le Wyoming. Jacques retirera de son séjour de précieux enseignements : une excellente maîtrise de l'anglais - ou de l'américain - que possédaient très peu d'ingénieurs français à cette époque ; et il avait de plus établi d'excellentes relations personnelles avec plusieurs de ses collègues, relations qui constitueront un de ses atouts au cours de sa carrière.

De retour en France il rejoignit le service Minerais que Lucien Coche avait installé à Saulnes et y reçut la responsabilité de deux domaines : la « réduction directe » (c'est-àdire hors du haut fourneau) et l'agglomération des minerais. Jacques Astier a joué dans ce dernier domaine un rôle majeur, tout d'abord en développant une cuve de simulation 
permettant d'établir les caractéristiques du procédé à l'échelle pilote, puis en amenant les responsables de la sidérurgie française, qui y étaient alors très réticents et considéraient cette technique comme coûteuse et sans grand intérêt, à adopter finalement de façon généralisée l'agglomération, provoquant ainsi une véritable révolution technique et économique dans l'élaboration de la fonte.

Jacques Astier n'a cessé d'enrichir sa documentation sur les sociétés métallurgiques et minières du monde entier et de cultiver les excellents rapports personnels qu'il avait établis avec un bon nombre de leurs techniciens et de leurs dirigeants. Aussi est-ce généralement par son intermédiaire que l'IRSID, qui avait acquis une solide expérience dans le domaine de la préparation des minerais, fut sollicité pour étudier tel ou tel de leurs problèmes spécifiques dans le cadre d'études sous contrat, opérations bienvenues du fait des ressources financières qu'elles apportaient. Et c'est naturellement Jacques Astier qui était chargé de gérer ces contrats. Cela lui imposait souvent de se déplacer et contribua à sa réputation justifiée de grand voyageur devant l'Éternel. Parmi les exemples de telles études, on peut citer celle sur la valeur du gisement saharien de Gara-Djebilet, ou celle confiée à l'IRSID par le gouvernement Iranien au début des années 60 : il s'agissait de bâtir un projet complet d'usine sidérurgique, sur des ressources minérales (minerai et charbon) à définir et dans un lieu à déterminer ; l'avant-projet établi au cours de cette étude a été entièrement réalisé, les mines proposées mises en exploitation et l'usine bâtie - par les russes - sous la forme et à l'endroit prévus ; l'IRSID est resté conseil des iraniens lors de la mise en exploitation de l'usine et de son extension en 1972.

Mais ces contacts ont surtout fait connaître Jacques Astier comme un expert internationalement reconnu du traitement des minerais ; $c^{\prime}$ est ainsi qu'il sera très vite appelé à siéger au sein de l'International Mineral Processing Comitee (IMPC), organisateur des grands congrès internationaux faisant, tous les deux ans environ, le point de cette industrie dans le monde. Il présidera cette instance de 1968 à 1985, et sera responsable des 8 congrès organisés au cours de cette période, de Leningrad et Varsovie à Sao Paulo ou Toronto, pour finir à Cannes en 1985. Il sera en 2010 honoré par l'IMPC Service Award, qui n'avait été jusque là décerné qu'à une seule autre occasion. Cette activité entraînera Jacques Astier à voyager d'autant plus : les anciens se souviennent de ce numéro du Journal of Metals des années 70 qui rendait compte brièvement de deux colloques qui s'étaient déroulés le même week-end l'un à Moscou ou à Leningrad, l'autre quelque part aux Etats-Unis : il était possible de voir Jacques sur les deux photos accompagnant ces articles, à l'ouverture de l'un et aux conclusions de l'autre!

Les connaissances acquises par Jacques dans le domaine de techniques peu familières à la sidérurgie française - ainsi que les réseaux de correspondants qu'il avait su se créer et cultiver dans de nombreux pays - représentaient une richesse dont il fallait faire bénéficier l'industrie nationale, qu'il s'agisse de la sidérurgie elle-même ou des constructeurs de matériel spécialisé. C'est ainsi que furent créées en 1974 deux organismes qu'il fut chargé de présider et d'animer : d'une part la Société Française des Produits Préréduits, SFMP, pour maintenir une information permanente sur le développement des techniques de réduction du minerai hors de la voie classique du haut-fourneau et réagir à toute opportunité qui apparaitrait de leur application dans le contexte particulier de la sidérurgie française, et d'autre part COFRANSID, un groupement économique liant les sociétés sidérurgiques et les constructeurs français de matériel, et visant à apporter à ces derniers pour leurs propositions à l'exportation les connaissances acquises par les chercheurs et les exploitants cette structure était rendue nécessaire par une déplorable habitude prise par la sidérurgie de se défier par principe des constructeurs français, de ne pas les accepter comme fournisseurs et partant de ne pas entretenir avec eux de relations régulières.

Expertise et reconnaissance internationale continueront à accompagner Jacques au-delà de sa retraite de l'IRSID qu'il prendra en 1988 pour s'installer dans le Morvan, dans la propriété achetée à Brassy : il y débute alors une carrière d'ingénieur-conseil qu'il poursuivra jusqu'à ses derniers jours. Cette nouvelle carrière lui donnera d'autres occasions de perfec- 
tionner ses connaissance par de nombreux voyages, voire de connaître de nouveaux pays comme le Vietnam; il y oeuvrera notamment pour le compte d'organismes internationaux, comme le Battelle Institute ou ITM à Genève, ville où il résidera de nombreux mois, ou encore l'ONUDI à Vienne, sans oublier les missions qu'il continuera de remplir en France pour la Revue de l'Industrie Minérale, la Revue de Métallurgie, les Techniques de l'Ingénieur ou l'École des Mines de Paris, pour que son expérience et toutes les données collectées pendant sa longue carrière puissent rester utiles au-delà de lui-même.

C'est en pleine activité que la mort viendra le surprendre brutalement, puisqu'il rentrait de Paris où il venait, trois jours à peine auparavant, de tenir une conférence. Il nous laisse le témoignage d'une exigence scientifique portée sur la connaissance experte d'une discipline et de sa mise en valeur technique, capable d'en tirer les conséquences dans la plupart des situations économiques, fussent-elles les plus particulières. Il restera aussi un modèle de transfert de connaissance et de relations entre les hommes, où la modestie de son approche lui a permis de tisser des liens étroits avec des spécialistes de très nombreux pays qui, au-delà des relations professionnelles, étaient devenus des amis.

Jacques Astier était chevalier de la Légion d'Honneur.

Charles Roederer

Pierre Emery 